\title{
Agriculture erases climate-driven $\beta$-diversity in Neotropical bird communities
}

Running head: Agriculture erases climate-driven $\beta$-diversity

Daniel S. Karp ${ }^{1,2^{*}}$, Luke O. Frishkoff ${ }^{3}$, Alejandra Echeverri ${ }^{2}$ Jim Zook $^{4}$, Pedro Juárez ${ }^{5}$, and Kai M.A. Chan ${ }^{2}$

${ }^{1}$ Department of Fish, Wildlife, and Conservation Biology, University of California, Davis, CA, 95616

${ }^{2}$ Institute for Resources, Environment, and Sustainability, University of British Colombia, Vancouver, BC V6T 1Z4

${ }^{3}$ Department of Ecology and Evolutionary Biology, University of Toronto, Toronto, ON M5S $1 \mathrm{~A} 1$

${ }^{4}$ Unión de Ornitólogos de Costa Rica, Apartado 182-4200, Naranjo de Alajuela, Costa Rica

${ }^{5}$ Herbario Nacional de Costa Rica, Departamento de Historia Natural, Museo Nacional de Costa Rica, Apdo. 749-1000, San José, Costa Rica

*to whom correspondese should be addressed: e-mail: dkarp@ucdavis.edu, phone: 530-219-9868 Keywords: agro-ecology, bird, climate change, environmental gradient, homogenization, multiple stressors, tropical, turnover Type of paper: primary research article 


\begin{abstract}
Earth is experiencing multiple global changes that will, together, determine the fate of many species. Yet how biological communities respond to concurrent stressors at local to regional scales is largely unknown. In particular, understanding how local habitat conversion interacts with regional climate change to shape patterns in $\beta$-diversity-differences among sites in their species compositions - is critical to forecast communities in the Anthropocene. Here, we study patterns in bird $\beta$-diversity across land-use and precipitation gradients in Costa Rica. We mapped forest cover, modeled regional precipitation, and collected data on bird community composition, vegetation structure, and tree diversity across 120 sites on 20 farms to answer three questions. First, do bird communities respond more strongly to changes in land-use or climate in northwest Costa Rica? Second, does habitat conversion eliminate $\beta$-diversity across climate gradients? Third, does regional climate control how communities respond to habitat conversion and, if so, how? After correcting for imperfect detection, we found that local land-use determined community shifts along the climate gradient. In forests, bird communities were distinct between sites that differed in vegetation structure or precipitation. In agriculture, however, vegetation structure was more uniform, contributing to $7-11 \%$ less bird turnover than in forests. Additionally, bird responses to agriculture and climate were linked: agricultural communities across the precipitation gradient shared more species with dry than wet forest communities. These findings suggest that habitat conversion and anticipated climate drying will act together to exacerbate biotic homogenization.
\end{abstract}




\section{Introduction}

The fate of Earth's wildlife will depend on species' collective responses to multiple global changes (Brook et al., 2008), yet most research focuses on how single pressures affect ecosystems. Moreover, interactive impacts of global change drivers are usually only considered locally (Mantyka-Pringle et al., 2012). Predicting biodiversity impacts at regional scales $(\gamma-$ diversity) hinges on understanding how species composition varies across space ( $\beta$-diversity) (Whittaker, 1960). $\beta$-diversity, however, is rarely considered in conservation planning, despite increasing evidence that biodiversity loss accumulates at larger scales (Socolar et al., 2016). For example, one 29-yr study revealed that climate change is homogenizing North Atlantic marine fish communities at a regional scale, but no temporal trends in local species richness were observed (Magurran et al., 2015). Indeed, biotic homogenization, and not local species loss, is increasingly recognized as a hallmark of the Anthropocene (McKinney \& Lockwood, 1999; Socolar et al., 2016).

Anthropogenic disturbances affect $\beta$-diversity through multiple processes. First, when disturbances decrease species richness, $\beta$-diversity can increase because the probability that sites do not share species increases when fewer species occupy each site (Chase et al., 2011). Second, disturbances can impose similar ecological filters over vast areas (Keddy, 1992), thereby homogenizing communities (Karp et al., 2012), or create environmental heterogeneity, thereby diversifying communities (Hawkins et al., 2015). These processes mediate the $\beta$-diversity impacts of two primary threats to terrestrial communities - habitat conversion and climate change. Habitat conversion often decreases $\beta$-diversity (Karp et al., 2012; Solar et al., 2015; Gossner et al., 2016) because it imposes ecological filters (Püttker et al., 2015) and homogenizes habitat structure across environmental gradients (Karp et al., 2012). However, the low $\alpha$ - 
diversity that follows habitat conversion can increase $\beta$-diversity through sampling effects (Karp et al., 2012). Indeed, a recent synthesis across 14,519 sites did not find anthropogenic habitats to consistently decrease $\beta$-diversity (Newbold et al., 2016). Though less studied than habitat conversion, climate change can also decrease $\beta$-diversity by favouring widely distributed species, often homogenizing environmental gradients, and because even if new species spread, colonization is not instantaneous (Britton et al., 2009; Davey et al., 2013; Magurran et al., 2015).

While habitat conversion and climate change may depress $\beta$-diversity in isolation (Socolar et al., 2016), little work has evaluated interactions between them (but see Holting et al., 2016). Individuals and populations regularly exhibit complex and non-additive responses to multiple stressors. For example, experiments (Mora et al., 2009), theory (Travis, 2003), and observations (Mantyka-Pringle et al., 2012) suggest that climate change and habitat conversion synergistically depress population sizes. Climate change and habitat conversion may also interactively determine community structure. Indeed, analyses of species' ranges suggests species' responses to climate and land-use are not independent. Hot (Clavero et al., 2011; Barnagaud et al., 2013) and dry-associated (Frishkoff et al., 2016) species are more likely to occupy agriculture than forests, meaning climate change and habitat conversion may shift community composition in similar ways. But the degree to which broad (species-range level) patterns predict local community shifts is unknown.

Here, we explore the combined impacts of climate drying and habitat conversion on birds in northwest Costa Rica, characterizing community change at 120 sites on 20 farms along steep independent land-use and precipitation gradients in a "space-for-time" substitution approach. Our focus is on precipitation because drying is predicted for northwest Costa Rica (Rauscher et $a l ., 2008)$ and prior work indicates that precipitation (not temperature) may drive bird responses 
to habitat conversion in Costa Rica (Frishkoff et al., 2016). We had three predictions. First, we hypothesized that species would sort along precipitation and land use gradients (Keil et al., 2012); however, turnover would be highest between land uses due to extreme differences in vegetation structure. Second, we predicted that communities exhibit more turnover in forest than agriculture because forests are heterogeneous, while agriculture is often structurally uniform, irrespective of location or regional climate (Flynn et al., 2009; Karp et al., 2012). Third, we predicted that agricultural vegetation is structurally similar to the shrubbier vegetation present in dry forests (Frishkoff et al., 2016); thus, agricultural communities should more closely resemble communities in drier than wetter habitats.

\section{Materials and Methods}

Study region and sites - To explore how land use and climate drying affect tropical bird communities, we hand-classified tree cover from aerial imagery, modeled regional precipitation from weather station data ( $\mathrm{N}=29$ stations), and surveyed bird and plant communities along orthogonal land use and precipitation gradients $(1500-3000 \mathrm{~mm})$ in northwest Costa Rica (the Guanacaste province; Fig. 1). This region's unique history has transformed it into a mosaic of protected areas, private forests, and agriculture. In the 1950s, beef production in Guanacaste rapidly increased, causing forest clearing (Calvo-Alvarado et al., 2009). Thirty years later, the industry collapsed, and forests regenerated, increasing in cover from $24 \%$ to $47 \%$ by 2005 (Calvo-Alvarado et al., 2009).

Guanacaste's climate is highly seasonal, with a severe dry season from December to April and a short, mid-rainy-season drought in July and August. It also has two rainy seasons, with the most intense rains occurring in October and November. Total annual precipitation varies from $\sim 1500 \mathrm{~mm}$ to $\sim 3000 \mathrm{~mm}$ over $\sim 75 \mathrm{~km}$, driving a shift from coastal wet forests to inland dry 
forests (Fig. S1). Yet Guanacaste's climate is changing. For several years, Guanacaste has been in severe drought, and climate projections suggest the region may experience up to $25 \%$ declines in summer precipitation over the next century (Rauscher et al., 2008).

We selected a network of 20 forest-adjoining farms across the regions' precipitation gradient including: pastures $(\mathrm{N}=12)$, rice $(\mathrm{N}=6)$, sugarcane $(\mathrm{N}=1)$, and Taiwan grass (a forage crop, $\mathrm{N}=1$ ) fields. Agricultural types were chosen to be representative of the broad study region. On each farm, we identified 6 sites to conduct bird point counts ( $N=120$ sites), half in agriculture and half in adjacent forests. Sites were chosen so that local land use (forest vs. agriculture) varied independently from landscape context (surrounding forest amount), with census points in forest interiors, forest edges, small forest fragments, agricultural field centers, fields bordering forest, and fields surrounded by forest. To measure surrounding forest cover, we hand-classified all tree cover within $1.5 \mathrm{~km}$ of point count locations using high-resolution, recent (2013-2016), cloud-free Google Earth images. The resulting tree-cover map was verified by using ground-truthed data from 480 points where we conducted vegetation surveys.

Farms varied strongly in total annual precipitation, but not in elevation or mean annual temperature: all farms were below 300m (mean: $110 \mathrm{~m}$; range: 0-300m), and experienced mean annual temperatures of $25-27^{\circ} \mathrm{C}$ (mean: $26^{\circ} \mathrm{C}$ ). Therefore, in this unique study system, effects of precipitation on bird communities can be isolated from effects due to temperature or elevation. Temperature data were acquired from WorldClim (www.worldclim.org/). Because validated precipitation maps were not available, we modeled spatial variation from weather station data collected between 1921 and 2015 and obtained from Costa Rica's Instituto Meteorológico Nacional (https://www.imn.ac.cr/; N=8), the Organization for Tropical Studies (http://www.ots.ac.cr/meteoro; N=1), KNMI Climate Explorer (https://climexp.knmi.nl/; N= 19), 
and one of our focal farms (http://tiopelon.cr/; $\mathrm{N}=1$ ). Data gaps were filled by averaging 2 previous and subsequent days (missing days) or by averaging the same month in 2 previous and subsequent years (missing months). Years with more than 3 months of missing data were omitted. To model regional precipitation, we used a general additive mixed model (GAMM) with a spatial thin-plate spline and 'Year' as a random intercept. Because offshore winds drive seasonal precipitation dynamics (Romero-Centeno et al., 2007), we also included distance from the coast to each census location along the predominant offshore wind direction as a covariate. A wind direction of $30^{\circ}$ North of West was chosen after consulting MERRA re-analysis data (http://globalwindatlas.com/). Our rainfall model predicted that the study sites spanned a range of $1500-2900 \mathrm{~mm}$ in annual precipitation.

Bird and vegetation surveys - In May-July of 2016, we surveyed all birds seen or heard in $20 \mathrm{~min}, 50 \mathrm{~m}$ fixed radius point counts. The same expert observer conducted all counts (J. Zook). Half of the point counts $(N=60)$ were repeatedly sampled $(3 x)$ within a one-week period and the other half were surveyed once so as to increase spatial replication while still allowing half the sites to be repeat sampled to estimate detection probabilities (see below). One farm (6 sites) was surveyed each day, beginning at sunrise and continuing for $\sim 5 \mathrm{hrs}$. Along with species identity, we also recorded the following abundance and detection covariates: time of day, date, ambient noise, number of people within the count radius, wind speed (using a handheld anemometer), and distance to nearest stream or river.

At each point count location, we also surveyed vegetation structure in four $5 \mathrm{~m}$-radius subplots, one at the point count center and the others $15 \mathrm{~m} \mathrm{~N}, 20 \mathrm{~m} \mathrm{SE}$, and $25 \mathrm{~m} \mathrm{SW}$ of plot center. First, we identified, marked, measured the diameter at breast height (DBH), and noted liana presence on all trees with $>5 \mathrm{~cm}$ DBHs. Second, for three canopy trees (or the next highest 
vegetation strata) at each subplot, we measured vegetation height (with a handheld hypsometer) and noted the presence of epiphytes or vines. Third, in two $1 \mathrm{~m}^{2}$ quadrats at each subplot, we recorded the number of woody stems and the percent coverage of shrubs and herbaceous vegetation. Fourth, we measured understory density by estimating (from $2 \mathrm{~m}$ away) the percent cover of meter stick, held $1.5 \mathrm{~m}$ from the ground. Finally, we analyzed fish-eye photographs at the center of each subplot with the software program 'Gap Light Analyzer' to quantify canopy cover.

Binomial-mixture model — We implemented a binomial mixture model to estimate species abundances at each site, while accounting for well-known variation in detection probability across species and sites (Royle \& Dorazio, 2008; Kéry \& Schaub, 2011). Specifically, the number of observed individuals $\left(Y_{i, j, k}\right)$ of each species $(i)$ at each site $(j)$ during each visit $(k)$ was assumed to result from detection and abundance processes such that:

$$
Y_{i, j, k} \sim \operatorname{Binomial}\left(\mathrm{N}_{i, j}, \mathrm{P}_{i, j, k}\right)
$$

Where $N$ is the true number of individuals, and $P$ is the detection probability of an individual. The detection process was modeled as:

$$
\begin{gathered}
\operatorname{logit}\left(\mathrm{P}_{i, j, k}\right)=\alpha 0_{L U[j]}+\alpha 1 * \text { time }_{j, k}+\alpha 2 * \text { noise }_{j, k}+\alpha 3 * p p l_{j, k}+\alpha 4 * \text { wind }_{j, k}+ \\
\theta 1_{i} * \text { date }_{j, k}+\delta 0_{i}+\delta 1_{i, j, k}
\end{gathered}
$$

Where $L U$ indicated whether a site was forested or not, time expressed time of day, noise was a dummy variable indicating whether load noises occurred during the count that could diminish sound-based detection of birds (e.g., farm equipment, cicadas, etc.), $p p l$ was the $(\log +1)$ number of farmworkers within the point count radius, wind was the wind speed at the beginning of the survey, and date was the Julian date that a survey took place. 
The true number of individuals $(N)$ was assumed to come from a Poisson distribution, based on the expected number of individuals $(\Lambda)$, which was modeled according to:

$$
\begin{gathered}
\log \left(\Lambda_{i, j}\right)=\beta 0_{i}+\beta 1_{i} * \operatorname{local}_{j}+\beta 2_{i} * \operatorname{precip}_{j}+\beta 3_{i} * \text { landscape }_{j, s}+ \\
\beta 4_{i} * \operatorname{local}_{j} * \operatorname{precip}_{j}+\beta 5_{i} * \operatorname{local}_{j} * \text { landscape }_{j, s}+\beta 6_{i} * \operatorname{precip}_{j} * \text { landscape }_{j, s}+ \\
\beta 7_{i} * \text { river }_{j}+\gamma 0_{i, j}+\gamma 1_{\left.i, \text { farm }_{[j}\right]}
\end{gathered}
$$

Above local describes the amount of forest cover within the $50 \mathrm{~m}$ radius point count, precip is the mean annual precipitation of the site, landscape is the landscape level tree cover at a biologically relevant spatial scale $(s)$ estimated from the data (see below), and river is the distance to the nearest river or stream.

Parameters in the $\alpha$ family were simple fixed effect terms. All parameters in the $\beta$ and $\theta$ families were estimated for each species, with species terms drawn from a normal distribution of mean $(\mu)$ and variance $\left(\sigma^{2}\right)$ estimated from the data. $\gamma$ and $\delta$ terms were random intercepts (variance estimated from data around a mean of 0 ) designed to incorporate additional variation for each species, site, or replicate that could not be explained by other fixed and random effects. Many taxa, including birds, respond not only local site conditions but also to the surrounding landscape, necessitating examination of abundance-responses at multiple spatial scales (Jackson \& Fahrig, 2015). We integrated over uncertainty regarding the 'correct' spatial scale directly within the binomial-mixture model (Frishkoff et al. 2017). Variable landscape $e_{j, s}$ was built from the 5m-resolution, hand-classified tree cover map. It consisted of a site-by-scale matrix filled with (centered and scaled) proportions of each site covered by trees within $60 \mathrm{~m}$ to $1.5 \mathrm{~km}$ radii (after removing the core $50 \mathrm{~m}$ radius, since this is considered as the 'local' land use). During the fitting process, the model evaluated alternative values of $s$, thereby gauging how well the full set of alternative spatial scales described the data. This resulted in a posterior distribution 
for values of $s$, which fully integrated over the uncertainly regarding the proper spatial scale, and which further can be used to select the most appropriate spatial scale (posterior mode) or an interval of spatial scales that well describe the data (credible interval).

All terms were assumed to be independent of other terms, with the exception of $\beta 1$ and $\beta 2$. Because we hypothesized that a species response to climate might be correlated to how it responds to local land-use change, we allowed the degree of correlation in these terms to be estimated from the data. Specifically:

$$
\left[\begin{array}{l}
\beta 1_{i} \\
\beta 2_{i}
\end{array}\right] \sim \operatorname{Norm}\left(\left[\begin{array}{l}
\mu_{\beta 1} \\
\mu_{\beta 2}
\end{array}\right],\left[\begin{array}{cc}
\sigma_{\beta 1}^{2} & \rho \sigma_{\beta 1} \sigma_{\beta 2} \\
\rho \sigma_{\beta 1} \sigma_{\beta 2} & \sigma_{\beta 2}^{2}
\end{array}\right]\right)
$$

Where $\rho$ represents the degree of correlation between a species land-use and precipitation response, and $\mu$ and $\sigma$ terms are the means and standard deviations estimated from the data.

We validated that our model was able to adequately describe the data using posterior predictive checks (Gelman \& Hill, 2007; Kéry \& Schaub, 2011). Briefly, along each iteration of the MCMC, we simulated a dataset under the parameter estimates in the model. We then compared the discrepancy between both the real data and the expectation based on parameter estimates, as well as the simulated data and the expectation based on parameter estimates using a chi-sq discrepancy statistic. These statistics were used to calculate a Bayesian p-value, describing whether the model was unlikely to generate the real data. Values $>0.05$ and $<0.95$ are taken to indicate adequate model fit (Kéry \& Schaub, 2011). Standard posterior predictive checks may be overly optimistic with regards to the ability of the model to describe the data, with Bayesian pvalues biased away from the tails (Marshall \& Spiegelhalter, 2003). These predictive checks should therefore be viewed primarily to indicate that there is nothing extremely incorrect about the model, though more subtle misspecifications may still exist. 
To quantify $\beta$-diversity we extracted $\mathrm{N}_{\mathrm{i}, \mathrm{j}}$ along each iteration of the posterior (hereafter referred to as 'posterior communities'). Each posterior community represents a possible number of true individuals that conform to the number of observed individuals at each site, given the detection probabilities estimated as a function of the species, sites, and visits during which a survey was conducted.

Quantifying pairwise dissimilarity — Because many $\beta$-diversity metrics exist, each describing a distinct aspect of community turnover or variation, we used multiple strategies to quantify and analyze bird $\beta$-diversity (Anderson et al., 2011). First, for each of the 2000 posterior communities, we calculated dissimilarity in community composition between each pair of sites as proportions of unshared species (Sorenson Index) and unshared individuals (BrayCurtis Index). Such total dissimilarity values, however, confound two patterns (species turnover and nestedness) that result from distinct processes (species replacement and loss) (Baselga, 2010). Thus, we partitioned total dissimilarities into turnover and nestedness components (Baselga, 2010, 2013a).

To ensure our results were robust, we also implemented an alternative approach of using null models to remove effects of species loss on $\beta$-diversity (Chase et al., 2011). Our method followed (Ponisio et al., 2016). First, for each posterior community, we simulated 1000 null communities, constraining the total number of individuals and species observed at each site. To do so, we randomized a binary matrix with a quasi-swap algorithm that maintained column and row sums. Next, we added individuals until we reached the total number of birds present at each site, sampling individuals with probabilities reflecting species' relative abundances across all sites. Second, we calculated Sorenson and Bray-Curtis dissimilarity for each pair of sites in each 
null community. Finally, for each pair of sites, we quantified ' $\alpha$-diversity corrected ranks,' as the proportion of null communities with dissimilarities less than that of the observed community.

ק-diversity in forests and agriculture - We visualized community turnover with NonMetric Multidimensional Scaling (NMDS). Plots were produced from averaging total pairwise dissimilarity, the turnover component of dissimilarity, and $\alpha$-diversity corrected ranks across 2000 posterior communities. In each case, we used Permutational Multiple Analysis of Variance (PERMANOVA), with 'farm' as a blocking factor, to statistically evaluate the relative roles of land use (forest vs. agriculture) and annual precipitation in determining species turnover.

We then assessed $\beta$-diversity in forests versus agriculture using two methods. First, we compared multivariate dispersion between land-use types. Analyses were conducted on Sorenson and Bray-Curtis dissimilarity indices, their turnover and nestedness components, and $\alpha$-diversity corrected ranks. Specifically, for each dissimilarity index and posterior community, we calculated the multivariate distance from each site to the centroid of all sites in the same land-use class (Anderson et al., 2006). The effect of land-use class on mean centroid distance was analyzed with Linear Mixed Models (LMMs), weighted by the posterior variance of centroid distance estimates (Tingley \& Beissinger, 2013). 'Farm' was included as a random intercept, and significance was assessed through comparing models with and without the land-use predictor via likelihood ratio tests evaluated against a chi-square distribution (Zuur et al., 2009). Pairwise nestedness metrics were log-transformed to ensure normality and homoscedasticity of residuals. To ensure that spatial autocorrelation within and between farms did not influence our results, we repeated the binomial mixture modeling, and all post-hoc $\beta$-diversity analyses, after including an exponential decay spatial covariance structure with a nugget and excluding the farm-level random effect. Residual spatial autocorrelation was minor, and explicitly modeling it did not 
change any of our results. We therefore chose to present analyses without explicit spatial covariance structure, other than the random effect of farm.

Second, we compared multi-site Sorenson and Bray Curtis dissimilarity in forest versus agricultural sites. To do so, we examined the difference between either the total, nestedness, or turnover components of multi-site dissimilarity in agricultural sites from the same measures in forest sites for each posterior community (Baselga, 2013b, 2016). We tested significance by determining whether 95\% Bayesian Credible Intervals of multi-site dissimilarity differences encompassed 0 .

$\boldsymbol{\beta}$-diversity drivers - We tested whether geographic distance, precipitation differences, and/or turnover in vegetation structure explained variation in bird communities. First, we aggregated vegetation data across subplots. Then, we compared nested LMMs with likelihood ratio tests to determine how precipitation affected vegetation structure variables in forest sites and whether relationships between vegetation structure and precipitation differed in forest versus agriculture sites. Next, we calculated Gower dissimilarity between sites, equally weighing: (1) canopy cover, (2) tree height, (3) ground cover composition (\% shrubs, \% herbaceous plants, \& number of woody stems), (4) understory density, (5) parasitic plant prevalence (number of trees with lianas, proportion of trees with epiphytes or vines), (6) number of tree stems, (7) average stem DBH, and (8) tree species richness. To compare variation in vegetation structure between forest and agriculture, we used Gower dissimilarities to calculate distances from each site to the centroid of its land-use class, and implemented LMMs to evaluate effects of land use on centroid distance (as above). We then used Mantel tests to examine associations between pairwise dissimilarities in vegetation structure, precipitation, and geographic distance in agriculture and forest. 
Next, we used Mantel tests to examine the correlation between pairwise community dissimilarity and dissimilarity in vegetation structure, differences in annual precipitation, and geographic distance between pairs of forest sites. First, for each metric of community dissimilarity, we calculated the median community dissimilarity between each pair of sites across the 2000 posterior communities. After separating forest and agricultural sites, we implemented Mantel tests to examine associations between community dissimilarity and environmental variables in forests and in agriculture. For vegetation and precipitation analyses, we also implemented Partial Mantel tests that included a geographic distance covariate to ensure that geographic distance alone could not explain our findings.

We then used a randomization procedure to assess the relative strength of community turnover in forest versus agricultural sites. To do so, we first created a matrix (hereafter termed the land-use comparison matrix) that codified the identity of each pair of sites as: (1) both sites in agriculture, (2) both sites in forest, or (3) one site in forest and one in agriculture. Second, for each environmental variable (vegetation structure, precipitation, and geographic distance), we constructed a simple linear model that had bird community dissimilarity as the response variable and the focal environmental variable, land-use comparison type, and their interaction as predictors. We parameterized these models such that the interaction term corresponded to the difference in the effect of each environmental variable on bird community dissimilarity between forest and agricultural sites. Specifically, linear models took the form:

$$
\text { ComDist }[\mathrm{i}]=\beta_{0, \text { comparison[i] }}+\beta_{1, \text { comparison[i] }}{ }^{*} \text { EnvDis }[\mathrm{i}]
$$

Where 'ComDist' is the community dissimilarity between a pair of sites (i), 'EnvDis' is the environmental distance between sites, and 'comparison' is the vectorized comparison matrix indicating pairwise site identity. Finally, we used permutation procedures to build null 
distributions for the difference between slope terms in agricultural comparisons versus forest comparisons (i.e., difference between $\beta_{1 \text {,agri-agri }}$ and $\beta_{1 \text {,for-for }}$ ). To confirm the robustness of our findings we used two procedures: (1) we permuted only the comparison matrix associated with $\beta_{1}$, so as to test the specific hypothesis that slopes differed, while holding comparison intercepts constant and (2) we calculated a null distribution by permuting the bird community dissimilarity matrix. Both approaches yielded qualitatively identical results, and we chose to present the more conservative test (permuting the land-use identity matrix).

Land use effects along climate gradients - To assess interactive impacts of climate drying and habitat conversion, we compared forest and agricultural communities along the precipitation gradient. First, to assess overall differences of forest communities to agriculture, we calculated the distance from each forest site to the centroid of all agricultural sites for each posterior community. Second, to examine community shifts between forest and agriculture at the individual farm level, we calculated the distance from each site to the centroid of the 3 sites in opposite land use within the same farm. Distances were calculated from Sorenson and BrayCurtis dissimilarities, their turnover components, and $\alpha$-diversity corrected ranks. To test whether the average agricultural community was more similar to forest communities in wetter or drier regions, we used LMMs to quantify effects of forest-site precipitation on the average distance to the agricultural centroid(s). To test whether precipitation affected the difference between agricultural and forest communities, we used LMMs to assess how the precipitation a farm experiences affects the dissimilarity between its forest and agricultural bird communities. In both cases, LMMs were weighted by the posterior variance of centroid distance estimates (Tingley \& Beissinger, 2013), and 'Farm' was included as a random intercept.

\section{Results}


Across all bird surveys, we detected 3813 individuals across 126 species (Forests: 2447 individuals, 100 species; Agriculture: 1366 individuals, 91 species). Our binomial-mixture model indicated that detection was not equal across sites, species, or replicate site visits. Birds were easier to detect in forest (mean across species of individual detection probability $22.6 \%$ [BCI: $18.8 \%-26.8 \%])$ than in agriculture (8.8\% [BCI: 6.5\%-11.5\%]). Detection was also easier in quieter environments (Noise effect term (logit scale), $-0.725[-1.047,-0.387])$ and earlier in the morning (Time effect, $-0.284[-0.392,-0.184])$. After accounting for detection, we inferred that an average of 99 individuals [95\% BCI: 74-142] and 32 species [30-36] used each 50m-radius forest point count, compared to 185 individuals [113-331] and 33 species [29-38] in agriculture. Posterior predictive checks indicated that models adequately described the data (Bayesian $p$ value $=0.46)$

$\boldsymbol{\beta}$-diversity along environmental gradients-Analyses of every $\beta$-diversity metric confirmed that bird communities exhibit turnover along the land-use and precipitation gradients (Fig. 2; Fig. S2). This trend was not driven by waterbirds, as they were no more abundant in wetter areas near the coast than in inland, drier areas (Fig. S3). Models indicated, however, that land-use explained more variation in bird community turnover than precipitation (Table S1).

$\boldsymbol{\beta}$-diversity within habitat types - Comparisons of the amount of $\beta$-diversity present within forest versus agriculture were metric-dependent. We predicted that forest sites would have greater $\beta$-diversity (as measured by distances to multivariate habitat centroid) than agricultural sites. Yet no differences in total $\beta$-diversity were observed within forest or agriculture when distances to group centroids were measured with total dissimilarity values (Fig. 3a/b; Table S2). Partitioning total $\beta$-diversity, however, showed that the turnover component of $\beta$-diversity was greater in forest $(P<0.01)$, while the nestedness component was higher in agriculture $(P<0.01$; 
Fig. 3c/d/e/f; Table S2). The same trend was observed after multisite dissimilarity metrics were analyzed rather than centroid distances (Fig. S4). When null models were used to factor out effects of $\alpha$-diversity, $\beta$-diversity was higher in forests than agriculture for incidence-based but not abundance-based dissimilarity (Fig. S5; Table S2).

Drivers of $\beta$-diversity across the precipitation gradient - Agriculture had more homogenous vegetation structure than forest, which may be one explanation for its lower turnover. Indeed, forest sites were on average 3 times more differentiated from one another in terms of vegetation structure than were agricultural sites $\left(\chi^{2}=71.6, P<0.001\right.$; Fig. S6, S7). Results were generally consistent when vegetation structure variables were log transformed, though agricultural plots with at least one tree were more strongly differentiated from those with no trees (Fig S6). Moreover, in agriculture, vegetation structure exhibited neither spatial patterns (Fig S8; Mantel: $\mathrm{r}=0.01, P=0.31$ ) nor changes along the precipitation gradient (Fig S8; Mantel: $\mathrm{r}=-0.04$, $P=0.82)$. But in forests, more distinct vegetation structure was observed between sites that were more distant (Fig S8; $\mathrm{r}=0.1, P=0.004$ ) and had more divergent precipitation regimes (Fig. S8; $\mathrm{r}=0.1, P=0.01)$. Specifically, compared to wetter sites, drier forests had marginally lower canopy cover and significantly shorter canopies, more herbaceous plant coverage, fewer woody stems, fewer trees with vines and epiphytes, and smaller tree diameters at breast height (Table S3). As a result, vegetation structure in agriculture was significantly more similar to vegetation structure in drier forest sites than wetter forest sites $\left(\chi^{2}=4.7, \mathrm{P}=0.03\right)$.

Forest bird communities tracked changes in vegetation structure, precipitation, and geographic distance. Specifically, all measures of incidence-based dissimilarity of bird communities increased between forest sites that were more distant and differed more strongly in vegetation structure and precipitation (Fig. 4: Table S4). Associations between incidence-based 
dissimilarity and vegetation/precipitation dissimilarity were also significant after accounting for co-variation with geographic distance (Table S4). In agriculture, however, relationships between incidence-based dissimilarity and vegetation structure, precipitation, and geographic distance were significantly weaker than in forest (Fig. 4). Agricultural bird communities never exhibited significantly increasing turnover with vegetation structure (Fig. 4), and also did not change along the precipitation gradient after partialing out geographic distance (Table S4). When abundancebased dissimilarity (Bray-Curtis) was used in each analysis, trends were qualitatively similar, but less pronounced and in some cases not significant (Table S4). Also, permutation tests yielded no support that abundance-based community turnover along vegetation, precipitation, or distance gradients was stronger in forest than in agriculture (Table S5).

Interactions between climate drying and habitat conversion - We observed a strong, significant correlation between species' abundance responses to habitat conversion and climate drying (Fig. 5). That is, species that thrived in forests tended to be more abundant in wet regions, while species that benefited from agriculture tended to reach maximal abundances in drier regions. As a result, the average agricultural community was more similar to forest communities in drier sites (significant in 6 of all 6 analyses, regardless of dissimilarity metric, whether $\beta$ diversity was partitioned, or if null models were used to account for $\alpha$-diversity; Table S4; Fig. 6, S9). Similarly, increasing precipitation tended to accentuate differences between forest and agricultural communities (Table S5; Fig. 6, S10). Specifically, bird communities located in forest and agricultural sites on the same farm were most distinct in wetter regions but became more similar in drier regions. This trend's direction was the same regardless of dissimilarity metric, partitioning strategy, or null-model implementation, and was significant in 4 of 6 analyses.

\section{Discussion}


Aligning with our first prediction, we found that bird community composition changes along precipitation and land-use gradients. While species sorting along climatic and habitat gradients is well documented (Mendenhall et al., 2016; Elsen et al., 2017), few studies have assessed community turnover along both gradients simultaneously (Keil et al., 2012). Our work highlights the relatively large impact that land-use change generates compared to regional climatic gradients in northwest Costa Rica (Table S1). However, land use influenced alternative components of $\beta$-diversity differently: while total $\beta$-diversity did not differ, the turnover component of $\beta$-diversity was higher in forests while the nestedness component was higher in agriculture. Because species richness was more variable among agricultural than forest sites (Fig. S11), higher nestedness in agriculture suggests that either consistent loss of particular species, and/or sampling effects that accompany random species loss, play a stronger role in assembling agricultural than forest communities (Karp et al., 2012).

Similarly, several explanations exist for why forest had higher turnover rates across the climate gradient. One is that agriculture is a strong filter, favoring a characteristic set of closelyrelated species with a limited suite of functional traits (Frishkoff et al., 2014; Gámez-Virués et al., 2015). However, we also found evidence for our second prediction that more heterogeneous vegetation structure in forests contributed to higher bird community turnover. In forests, bird communities tracked changes in vegetation structure, precipitation, and geographic distance. In agriculture, vegetation structure was more uniform (as in Flynn et al., 2009), and did not influence bird turnover. Also unlike in forests, agricultural vegetation exhibited no significant changes across the precipitation gradient. This may explain why forest but not agricultural bird communities still exhibited turnover along the precipitation gradient after including distance covariates (Table S3). Indeed, when comparing sites with similar precipitation, communities 
exhibited equivalent turnover in forest versus agriculture; however, community turnover between sites on opposite ends of the precipitation gradient was $13 \%$ higher in forests than in agriculture.

Evidence that vegetation structure mediates climatic effects on birds is accumulating (Jankowski et al., 2009, 2013; Ferger et al., 2014; Bennett et al., 2015), but a mechanistic understanding for the relationship remains elusive. Effects may be direct if climate-induced vegetation changes decrease nesting, foraging, or roosting site availability. In Australia, for example, prolonged drought caused dramatic changes in vegetation structure and in bird communities, especially in smaller forest fragments (Bennett et al., 2015). Ground and burrowing nesters exhibited the strongest declines, which may have been due to reductions in shrub and herbaceous ground cover that may serve as nesting sites (Bennett et al., 2015).

Climate-induced changes in vegetation structure may also affect bird communities indirectly. First, changes in vegetation structure may decrease food availability. For example, warmer and wetter climates in Tanzania were shown to increase vegetation heterogeneity, which bolstered fruit and invertebrate food resources, which in turn increased frugivore and insectivore richness (Ferger et al., 2014). In Panama, longer dry seasons decreased bird population growth rates and recruitment, most notably for fruit and seed eating species whose food resources are often sensitive to tropical precipitation dynamics (Brawn et al., 2016). Second, changing vegetation structure could impose new top-down controls on bird communities. In the United States, decreased snowfall caused declines in woody plant density, which exposed multiple songbird species to elevated nest predation rates (Martin \& Maron, 2012). Third, vegetation structure change could benefit a few strong competitors, altering competitive dynamics. In Australia, prolonged drought created more open habitats where Noisy Miners (Manorina melanacephala) thrived and displaced other native species (Bennett et al., 2015). Finally, 
climate-induced vegetation structure may alter local microclimates and impose new physiological filters on birds. Sites with open, "shrubby" vegetation, for example, may simply be too hot for some species to persist.

Regardless of mechanism, a strong relationship between birds and vegetation structure was likely responsible for our observation that bird communities exhibit non-independent responses to land-use and precipitation gradients. With shorter, thinner trees and more herbaceous plants, drier forests were more similar to agriculture in vegetation structure than wetter forests were to agriculture. As a result, species that were more common in agriculture tended to increase in abundance in drier regions, while more forest-affiliated species tended to increase in wetter regions. Thus, we found evidence for our third prediction: agricultural communities were, on average, more similar to the communities present in drier than wetter forests.

Importantly, some of the trends that we identified would not have been apparent had we analyzed raw detection data (as in most prior $\beta$-diversity studies), because of the large amount of detection error for any given sampling instance, and because of variable detectability between species and across habitats. Nonetheless, current methods for modeling detection are not without faults. First, for highly mobile organisms (such as birds) that are not bound to highly localized areas, such models estimate the number of animals that use a given area throughout the season, rather than a point abundance at a given time. Second, and more importantly, binomial mixture models may overestimate the abundance of rare species in the meta-community (Gomez et al., 2017), though this behavior is minimized as the number of sampled sites increases and the absolute magnitude of overestimation is fairly small. With respect to our findings, overestimation of rare species could either result in more or less apparent $\beta$-diversity depending on the manner 
and locations in which rare species are overestimated. Further methods development that corrects for model pathologies regarding rare species is clearly needed. Ultimately, however, we believe that modeling detection uncertainty is a substantial improvement over analyzing observed counts. Because detection is known to vary strongly across species and habitat types, implicitly assuming perfect detection can lead to false conclusions about occupancy and abundance trends across environmental gradients (e.g., Ruiz-Gutiérrez et al., 2010).

Across the tropics, agriculture is continuing to replace forests (Hansen et al., 2013), and, though climate models predict spatial variation in precipitation trends, drying is expected to intensify in northwest Costa Rica (Rauscher et al., 2008; Magrin et al., 2014). Our results provide the first direct evidence that species and community-level responses to both global changes may not be independent. Prior studies have shown (at a much coarser scale) that species' climate envelopes are predictive of their habitat preferences: species with ranges encompassing dry (Frishkoff et al., 2016) and hot (Clavero et al., 2011; Barnagaud et al., 2013) regions are more likely to thrive in agriculture than forests. Moreover, prior work suggests regional climate regimes may even determine habitat preferences, with agricultural species invading forests in regions where climates are warmer or vegetation structure is shorter and shrubbier (Frishkoff et al., 2015, 2016). Together, our work and these prior findings suggest that habitat conversion and climate drying may favor the same species and homogenize communities more rapidly than previously anticipated. Looking forward, our work thus suggests that wet forest bird communities across the tropics may be particularly vulnerable to global change. Because ongoing climate drying and deforestation appear to both push community composition in the similar direction, maintaining future community-level biodiversity will likely necessitate preserving wet-forest refugia and developing targeted conservation plans for wet-forest wildlife. 


\section{Acknowledgements}

We thank W. Anderegg, L. Anderegg, W. Ou X.M. Campos Palma, E. Rodríguez Paniagua, D. Steyn, and the rest of the FurturAgua team and staff of the Área de Conservación Tempisque for support with fieldwork, data compilation, and analysis. We also thank the many private landowners that provided us with access to their properties, and the local communities in Costa Rica for support. We are grateful for financial support through a Killam postdoctoral fellowship to DSK, a Doctoral Graduate Leadership Fellowship from UBC to AE, a University of Toronto EEB post-doctoral fellowship to LOF, and the Canada Research Chairs Program (KMAC). This work was also funded by a FuturAgua project grant from a Belmont Forum NSERC grant (\#G8PJ-437336-2012) and an NSERC Discovery Grant (UBC \# 06-5566). This study was conducted under the auspices of the University of British Columbia, Animal Care Committee approval number A15-0109, and Costa Rican government approval with research permits SINAC- SE-CUS-PI-R-036-2016.

\section{References}

Anderson MJ, Ellingsen KE, McArdle BH (2006) Multivariate dispersion as a measure of beta diversity. Ecology Letters, 9, 683-693.

Anderson MJ, Crist TO, Chase JM et al. (2011) Navigating the multiple meanings of $\beta$ diversity: a roadmap for the practicing ecologist. Ecology Letters, 14, 19-28.

Barnagaud J, Barbaro L, Hampe A, Jiguet F, Archaux F (2013) Species' thermal preferences affect forest bird communities along landscape and local scale habitat gradients. Ecography, 36, 1218-1226.

Baselga A (2010) Partitioning the turnover and nestedness components of beta diversity. Global Ecology and Biogeography, 19, 134-143. 
Baselga A (2013a) Separating the two components of abundance-based dissimilarity: Balanced changes in abundance vs. abundance gradients. Methods in Ecology and Evolution, 4, 552557.

Baselga A (2013b) Multiple site dissimilarity quantifies compositional heterogeneity among several sites, while average pairwise dissimilarity may be misleading. Ecography, 36, 124 128.

Baselga A (2016) Partitioning abundance based multiplesite dissimilarity into components: balanced variation in abundance and abundance gradients. Methods in Ecology and Evolution.

Bennett JM, Clarke RH, Horrocks GFB, Thomson JR, Mac Nally R (2015) Climate drying amplifies the effects of land-use change and interspecific interactions on birds. Landscape Ecology, 30, 2031-2043.

Brawn JD, Benson TJ, Stager M, Sly ND, Tarwater CE (2016) Impacts of changing rainfall regime on the demography of tropical birds. Nature Climate Change, 7, 133-136.

Britton AJ, Beale CM, Towers W, Hewison RL (2009) Biodiversity gains and losses: Evidence for homogenisation of Scottish alpine vegetation. Biological Conservation, 142, 1728-1739.

Brook BW, Sodhi NS, Bradshaw CJA (2008) Synergies among extinction drivers under global change. Trends in Ecology and Evolution, 23, 453-460.

Calvo-Alvarado J, McLennan B, Sanchez-Azofeifa A, Garvin T (2009) Deforestation and forest restoration in Guanacaste, Costa Rica: Putting conservation policies in context. Forest Ecology and Management, 258, 931-940.

Chase JM, Kraft NJB, Smith KG, Vellend M, Inouye BD (2011) Using null models to disentangle variation in community dissimilarity from variation in $\alpha$-diversity. Ecosphere, 2 , 
A24.

Clavero M, Villero D, Brotons L (2011) Climate change or land use dynamics: do we know what climate change indicators indicate? PloS One, 6, e18581.

Davey CM, Devictor V, Jonzén N, Lindström Å, Smith HG (2013) Impact of climate change on communities: revealing species' contribution. Journal of Animal Ecology, 82, 551-561.

Elsen PR, Tingley MW, Kalyanaraman R, Ramesh K, Wilcove DS (2017) The role of competition, ecotones, and temperature in the elevational distribution of Himalayan birds. Ecology, 98, 337-348.

Ferger SW, Schleuning M, Hemp A, Howell KM, Böhning-Gaese K (2014) Food resources and vegetation structure mediate climatic effects on species richness of birds. Global Ecology and Biogeography, 23, 541-549.

Flynn DFB, Gogal-Prokurat M, Molinari N et al. (2009) Loss of functional diversity under land use intensification across multiple taxa. Ecology Letters, 12, 22-33.

Frishkoff LO, Karp DS, M'Gonigle L et al. (2014) Loss of avian phylogenetic diversity in neotropical agricultural systems. Science, 345, 1343-1346.

Frishkoff LO, Hadly EA, Daily GC (2015) Thermal niche predicts tolerance to habitat conversion in tropical amphibians and reptiles. Global Change Biology, 21, 3901-3916.

Frishkoff LO, Karp DS, Flanders JR, Zook JR, Daily GC, M'Gonigle LK (2016) Climate change and habitat conversion favour the same species. Ecology Letters, 19, 1081-1090.

Frishkoff L.O., Mahler D.L., M.J. Fortin (2017) Integrating over uncertainty in spatial scale of response within multispecies occupancy models yields more accurate assessments of community composition. bioRxiv. https://doi.org/10.1101/143669

Gámez-Virués S, Perović DJ, Gossner MM et al. (2015) Landscape simplification filters species 
traits and drives biotic homogenization. Nature Communications, $\mathbf{6}, 8568$.

Gelman A, Hill J (2007) Data Analysis Using Regression and Multilevel/Hierarchical Models. Cambridge University Press, New York.

Gomez JP, Robinson SK, Ponciano JM (2017) An efficient extension of N-mixture models for multi-species abundance estimation. bioRxiv, 1-38.

Gossner MM, Lewinsohn TM, Kahl T et al. (2016) Land-use intensification causes multitrophic homogenization of grassland communities. Nature, 540, 266-269.

Hansen MC, Potapov P, Moore R et al. (2013) High-resolution global maps of 21st century forest cover change. Science, 850, 850-854.

Hawkins CP, Mykrä H, Oksanen J, Vander Laan JJ (2015) Environmental disturbance can increase beta diversity of stream macroinvertebrate assemblages. Global Ecology and Biogeography, 24, 483-494.

Holting M, Bovolo CI, Ernst R (2016) Facing complexity in tropical conservation: how reduced impact logging and climatic extremes affect beta diversity in tropical amphibian assemblages. Biotropica, 48, 528-536.

Jackson HB, Fahrig L (2015) Are ecologists conducting research at the optimal scale? Global Ecology and Biogeography, 24, 52-63.

Jankowski JE, Ciecka AL, Meyer NY, Rabenold KN (2009) Beta diversity along environmental gradients : implications of habitat specialization in tropical montane landscapes. Journal of Animal Ecology, 78, 315-327.

Jankowski JE, Merkord CL, Rios WF, Revilla NS, Silman MR, Garc1 K (2013) The relationship of tropical bird communities to tree species composition and vegetation structure along an Andean elevational gradient. Journal of Biogeography, 40, 950-962. 
Karp DS, Rominger AJ, Zook J, Ranganathan J, Ehrlich PR, Daily GC (2012) Intensive agriculture erodes $\beta$-diversity at large scales. Ecology Letters, 15, 963-970.

Keddy PA (1992) Assembly and response rules: two goals for predictive community ecology. Journal of Vegetation Science, 3, 157-164.

Keil P, Schweiger O, Kühn I et al. (2012) Patterns of beta diversity in Europe: The role of climate, land cover and distance across scales. Journal of Biogeography, 39, 1473-1486.

Kéry M, Schaub M (2011) Bayesian Population Analysis using WinBUGS - a hierarchical perspective. Academic Press.

Magrin GO, Marengo JA, Boulanger J-P et al. (2014) Central and South America. In: Climate Change 2014: Impacts, Adaptation, and Vulnerability. Part B: Regional Aspects. Contribution of Working Group II to the Fifth Assessment Report of the Intergovernmental Panel on Climate Change (eds Barros VR, Field CB, Dokken, D.J., Mastrandrea MD, Mach KJ, Bilir TE, Chatterjee M, Ebi KL, Estrada YO, Genova RC, Girma B, Kissel ES, Levy AN, MacCracken S, Mastrandrea PR, White LL), pp. 1499-1566. Cambridge University Press, Cambridge, UK.

Magurran AE, Dornelas M, Moyes F, Gotelli NJ, McGill B (2015) Rapid biotic homogenization of marine fish assemblages. Nature Communications, $\mathbf{6}, 8405$.

Mantyka-Pringle CS, Martin TG, Rhodes JR (2012) Interactions between climate and habitat loss effects on biodiversity: a systematic review and meta-analysis. Global Change Biology, 18, $1239-1252$.

Marshall EC, Spiegelhalter DJ (2003) Approximate cross-validatory predictive checks in disease mapping models. Statistics in Medicine, 22, 1649-1660.

Martin TE, Maron JL (2012) Climate impacts on bird and plant communities from altered animal 
- plant interactions. Nature Climate Change, 2, 195-200.

McKinney ML, Lockwood JL (1999) Biotic homogenization: a few winners replacing many losers in the next mass extinction. Trends in Ecology \& Evolution, 14, 450-453.

Mendenhall CD, Shields-Estrada A, Krishnaswami AJ, Daily GC (2016) Quantifying and sustaining biodiversity in tropical agricultural landscapes. Proceedings of the National Academy of Sciences, 113, 14544-14551.

Mora C, Metzger R, Rollo A, Myers RA (2009) Experimental simulations about the effects of overexploitation and habitat fragmentation on populations facing environmental warming. Proceedings of the Royal Society B, 274, 1023-1028.

Newbold T, Hudson LN, Hill SLL et al. (2016) Global patterns of terrestrial assemblage turnover within and among land uses. Ecography, 39, 1151-1163.

Ponisio LC, M'Gonigle LK, Kremen C (2016) On-farm habitat restoration counters biotic homogenization in intensively managed agriculture. Global Change Biology, 22, 704-715.

Püttker T, de Arruda Bueno A, Prado PI, Pardini R (2015) Ecological filtering or random extinction? Beta-diversity patterns and the importance of niche-based and neutral processes following habitat loss. Oikos, 124, 206-215.

Rauscher SA, Giorgi Æf, Diffenbaugh NS, Seth Æa (2008) Extension and intensification of the Meso-American mid-summer drought in the twenty-first century. Climate Dynamics, 31, $551-571$.

Romero-Centeno R, Zavala-Hidalgo J, Raga GB (2007) Midsummer gap winds and low-level circulation over the eastern tropical Pacific. Journal of Climate, 20, 3768-3784.

Royle JA, Dorazio RM (2008) Hierarchical Modeling and Inference in Ecology. Academic Press, London. 
Ruiz-Gutiérrez V, Zipkin EF, Dhondt A a. (2010) Occupancy dynamics in a tropical bird community: unexpectedly high forest use by birds classified as non-forest species. Journal of Applied Ecology, 47, 621-630.

Socolar JB, Gilroy JJ, Kunin WE, Edwards DP (2016) How should beta-diversity inform biodiversity conservation? Trends in Ecology and Evolution, 31, 67-80.

Solar RR de C, Barlow J, Ferreira J et al. (2015) How pervasive is biotic homogenization in human-modified tropical forest landscapes? Ecoloy Letters, 18, 1108-1118.

Tingley MW, Beissinger SR (2013) Cryptic loss of montane avian richness and high community turnover over 100 years. Ecology, 94, 598-609.

Travis JMJ (2003) Climate change and habitat destruction: a deadly anthropogenic cocktail. Proceedings of the Royal Society B, 270, 467-473.

Whittaker RH (1960) Vegetation of the Siskiyou Mountains, Oregon and California. Ecological Monographs, 30, 279-338.

Zuur AF, Ieno EN, Walker NJ, Saveliev AA, Smith GM (2009) Mixed effects models and extensions in ecology with $R$. Springer, New York. 


\section{Figures}

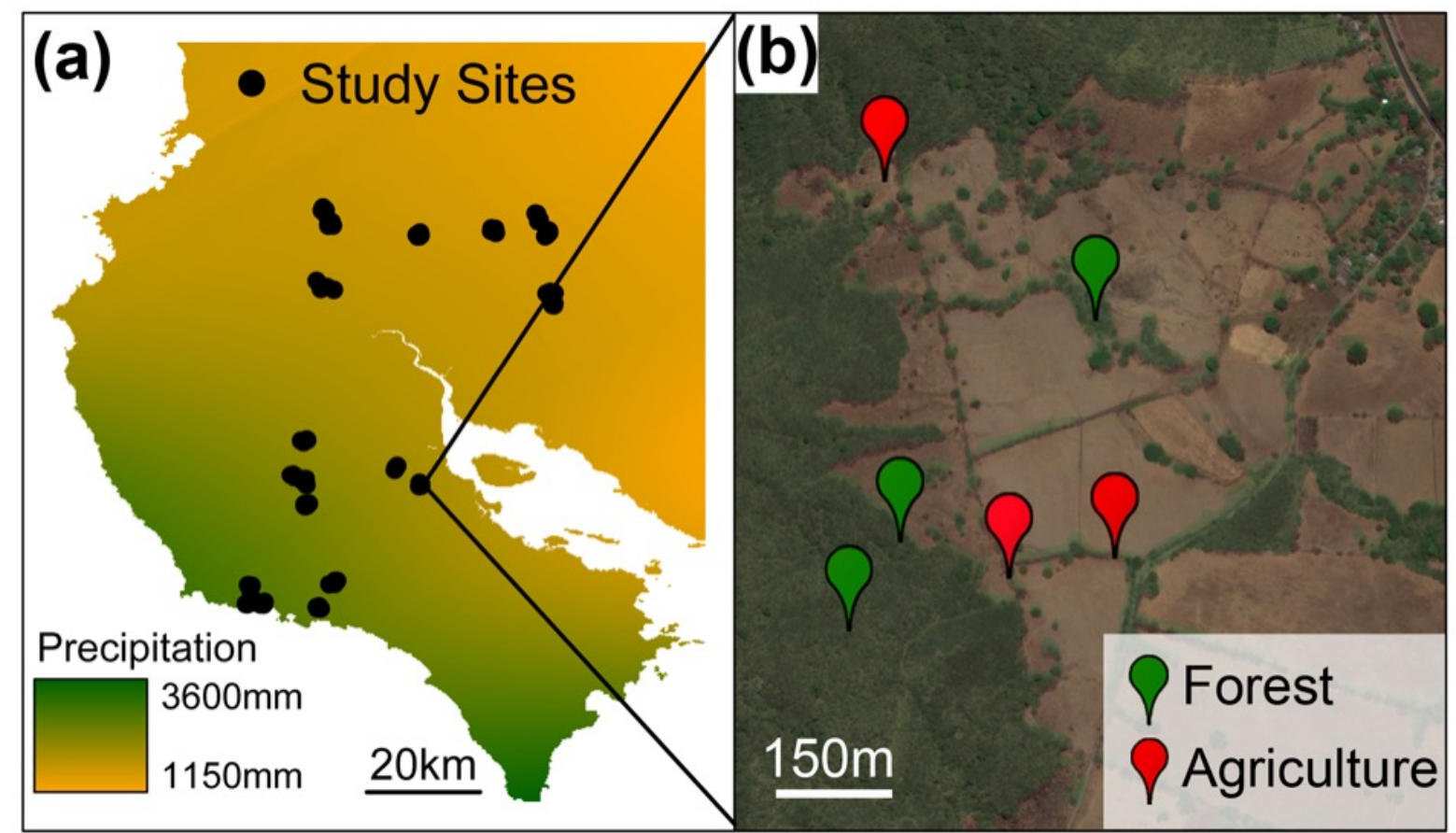

Figure 1: Map of study sites and precipitation gradient. Panel A shows modeled mean annual

precipitation across the study region, as well as study site locations (black dots). Sites were arrayed across a precipitation gradient. Panel B shows point count locations at an example farm. At each site, three point counts were placed in agriculture (red icons) and three in forest (green icons). Locations were chosen so that local land use and landscape context (proportion of surrounding tree cover; green shading) were largely independent. 

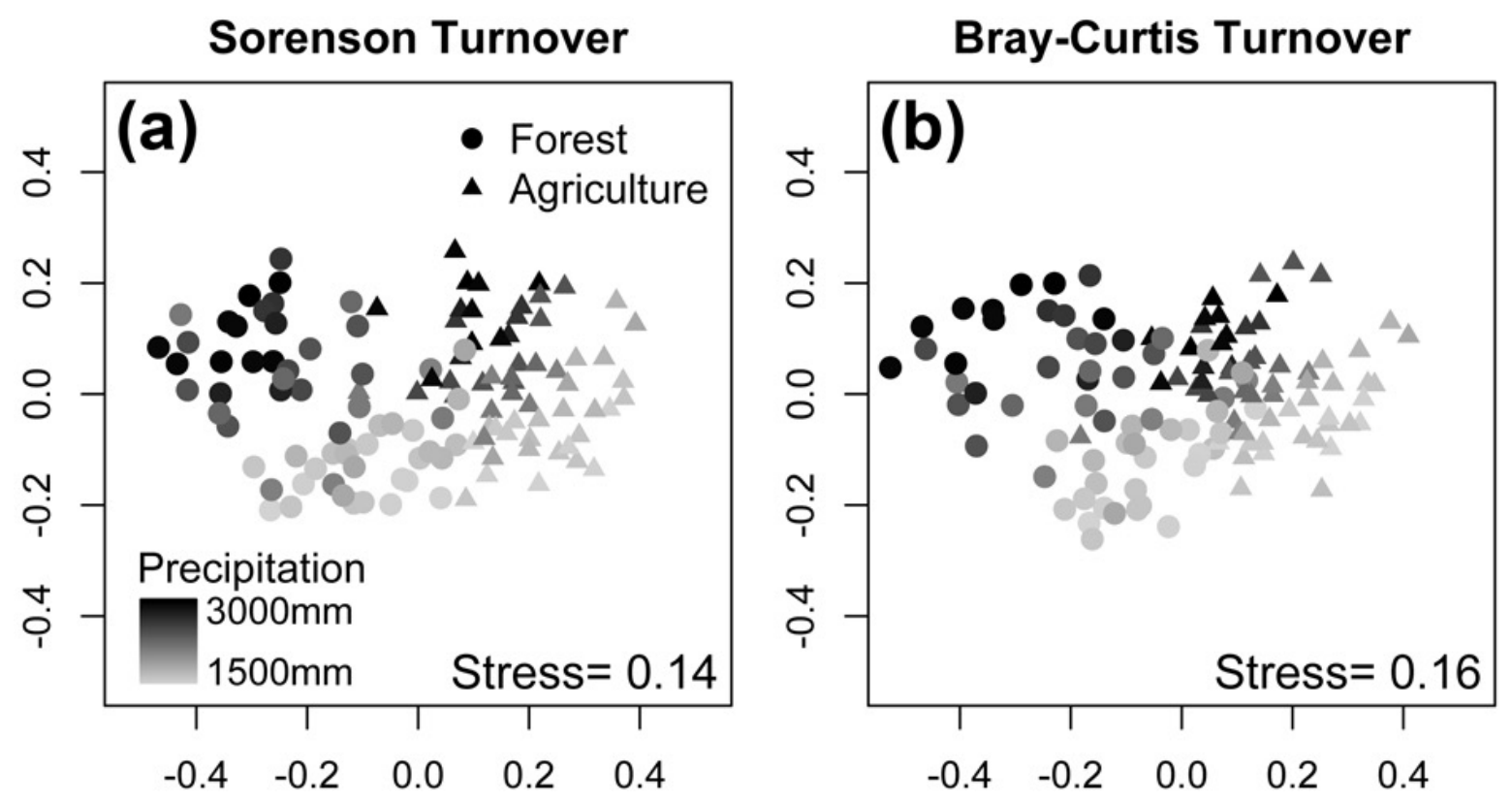

Figure 2: Non-metric multi-dimensional scaling (NMDS) plots depicting effects of land use and precipitation on bird communities. The distance between sites (points) represents distinctness in community composition, calculated as turnover in species (Sorenson, Panel A) or individuals (Bray-Curtis, Panel B). Axes represent ordination distances. Triangles are sites in agriculture, circles are in forest, and points are colored by precipitation. Plots show significant differences in bird composition between forests and agriculture and along the precipitation gradient (Table S1). 

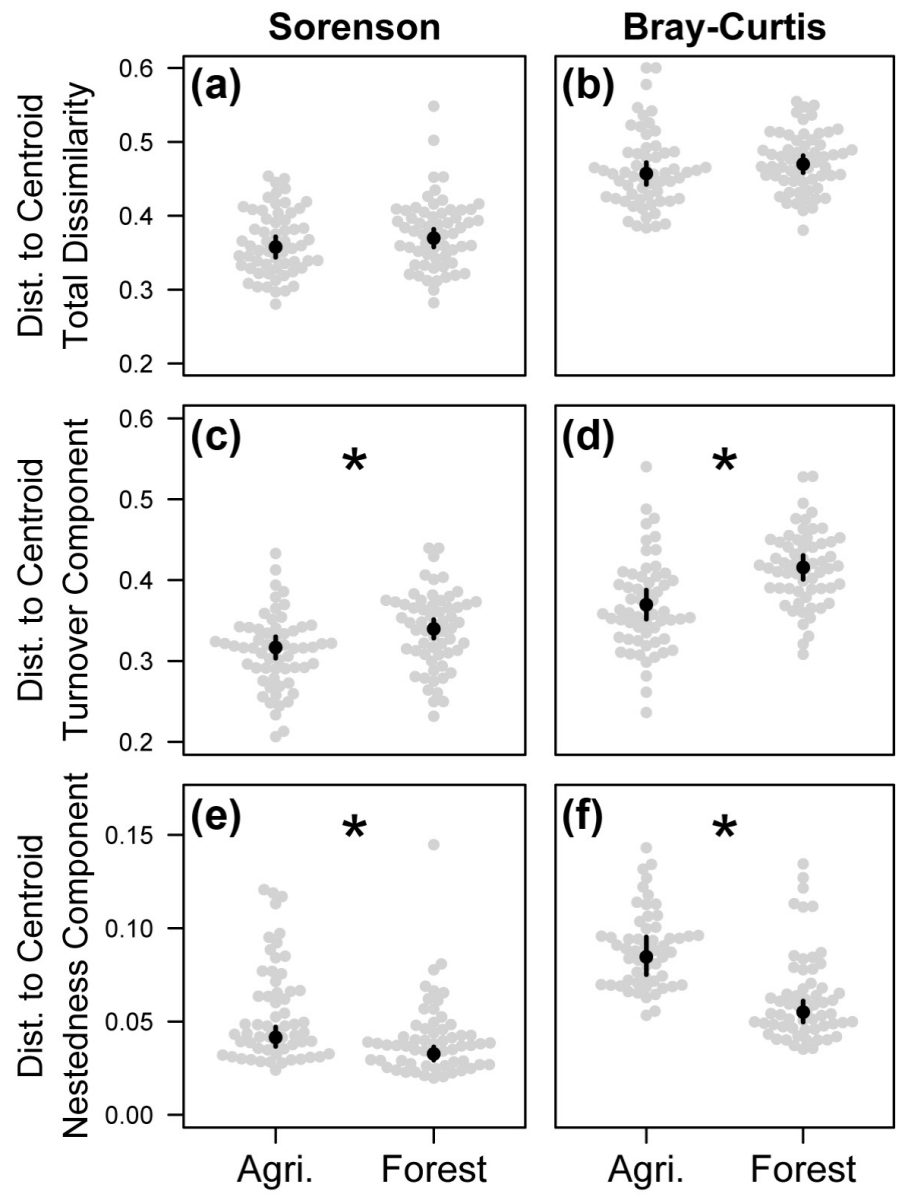

Figure 3: $\boldsymbol{\beta}$-diversity comparisons between forest and agriculture. Plots depict distances to group centroids (a measure of $\beta$-diversity) in agricultural and forest point count locations (dots). $\beta$-diversity is calculated as unshared species (left panels) and unshared individuals (right panels). No differences in total dissimilarity are observed between forest and agriculture (panels A and B). However, partitioning $\beta$-diversity into the components generated from species replacement (turnover) versus species loss (nestedness) reveals significantly more turnover in forest (panels C and D) and more nestedness in agricultural (panels E and F). Black dots are mean values; lines are $95 \%$ confidence intervals. Asterisks denote significance $(\mathrm{P}<0.05)$ under likelihood ratio tests, comparing nested generalized linear mixed models (GLMMs). 

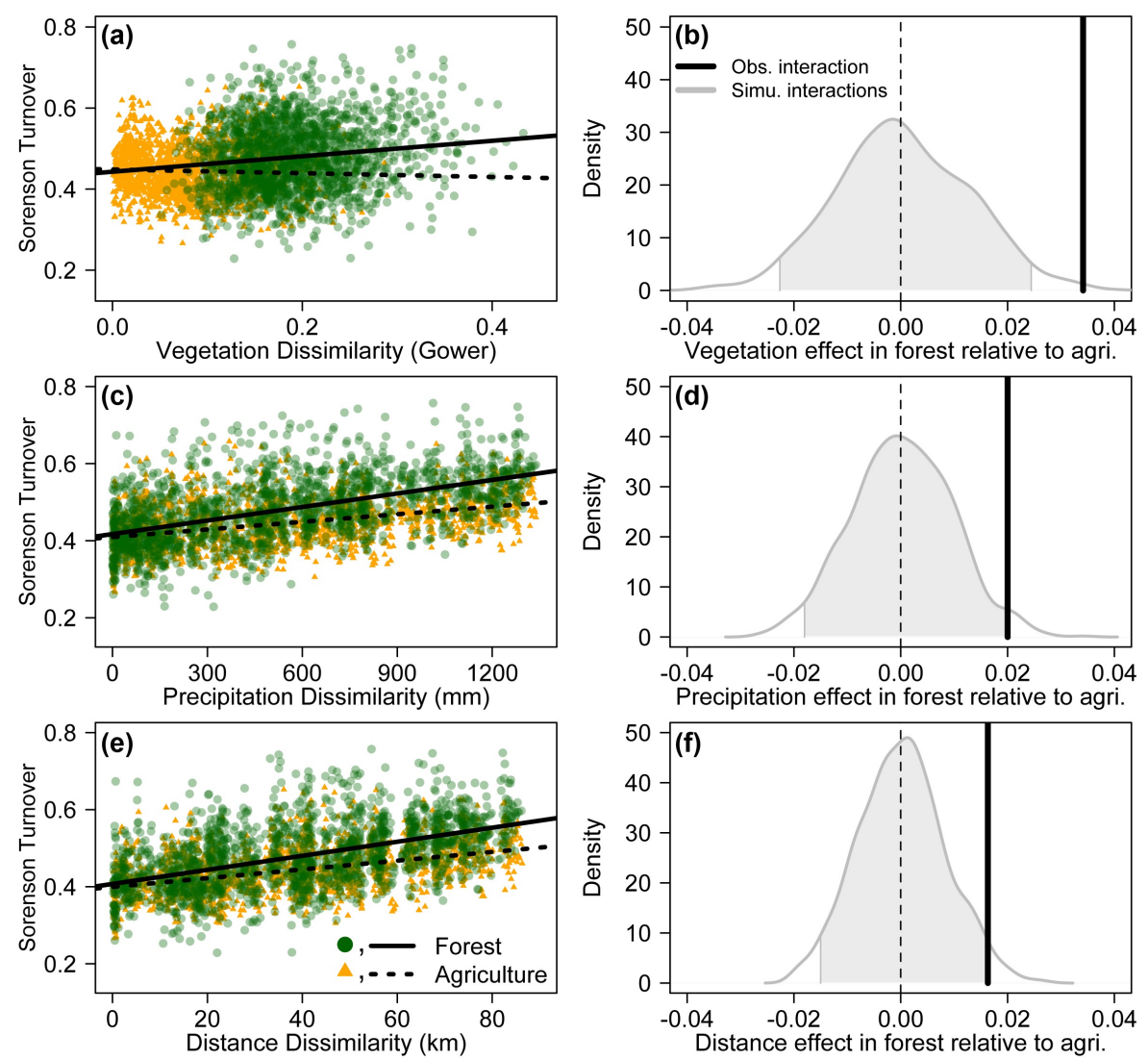

Figure 4: Patterns in bird community turnover along gradients of vegetation structure,

precipitation, and geographic distance. Differences in vegetation structure (Gower

dissimilarity; Panel A), precipitation (mm rainfall; Panel D), and distance (km; Panel G) between pairs of forest (green dots) and agricultural count locations (orange dots) are graphed against difference in community composition (the turnover component of Sorenson dissimilarity). Solid (forest) and dotted (agriculture) lines depict linear model relationships. Solid black lines in Panels B, D, and F are observed interactions between local land-use (forest versus agriculture) and each environmental gradient (equivalent to the difference in slopes of solid versus dotted lines in left panels). Gray histograms are density diagrams of interaction values across 1000 community randomizations (see methods). Plots show that communities exhibited stronger turnover along vegetation (Panel B), precipitation (D), and distance (F) gradients in forest sites than in agricultural sites. 


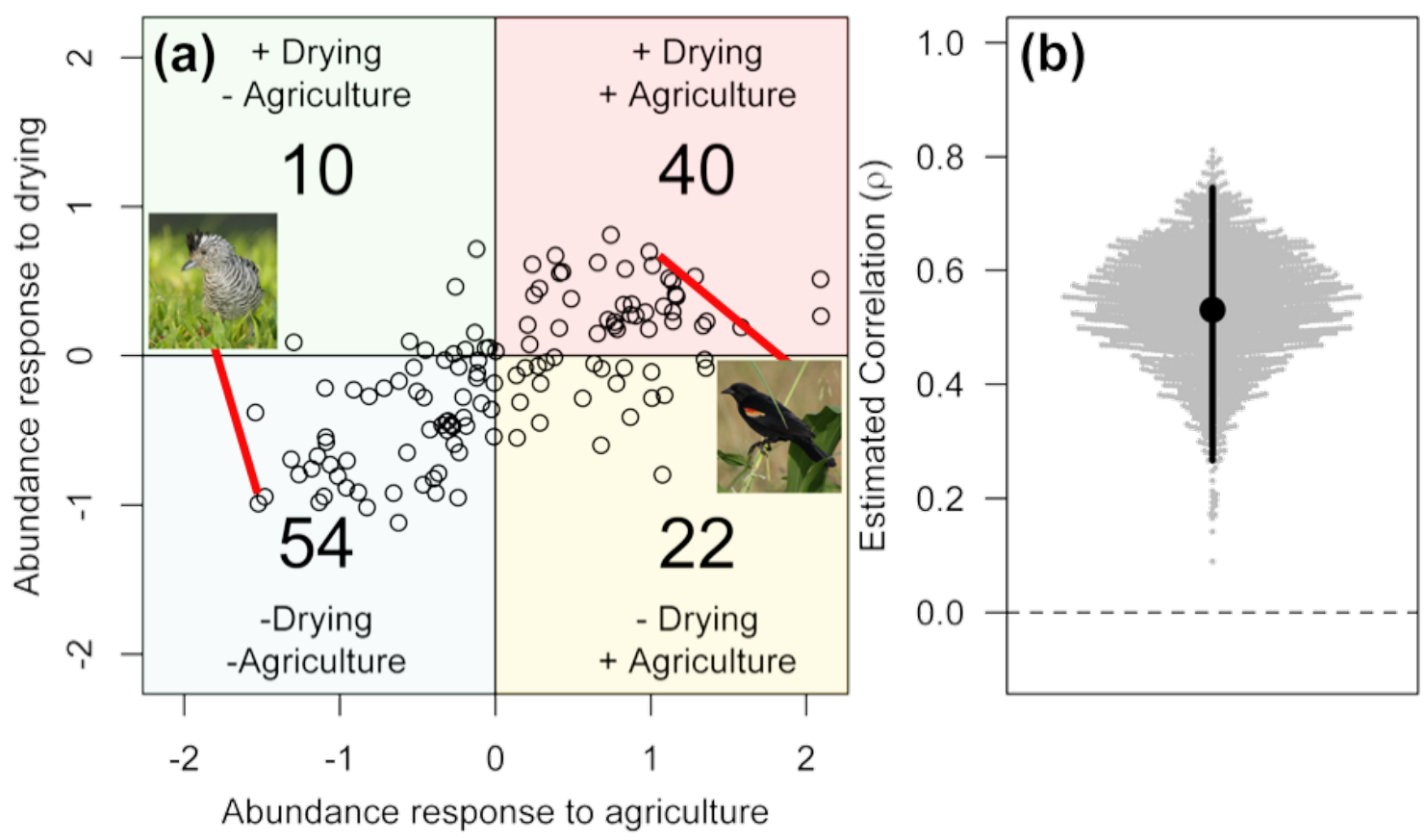

Figure 5: Correlated responses to habitat conversion and climate drying among

Neotropical birds. Panel A depicts responses of each species (dots) to agriculture and drying, estimated directly from binomial-mixture models. Numbers represent number of species predicted to increase or decrease in abundance in response to each stressor. Species that responded positively to agriculture also responded positively to drying (e.g., the pictured Redwinged Blackbird, Agelaius phoeniceus, identified with a red line) and species that were sensitive to habitat conversion were also sensitive to drying (e.g., Barred Antshrike, Thamnophilus doliatus). Panel B depicts the posterior distribution of the estimated correlation ( $\rho$ from the multivariate normal distribution) between species response to land-use and climate gradients (gray dots are 2000 posterior samples). Black dot is the correlation; line is the $95 \%$ Bayesian Credible Interval. 

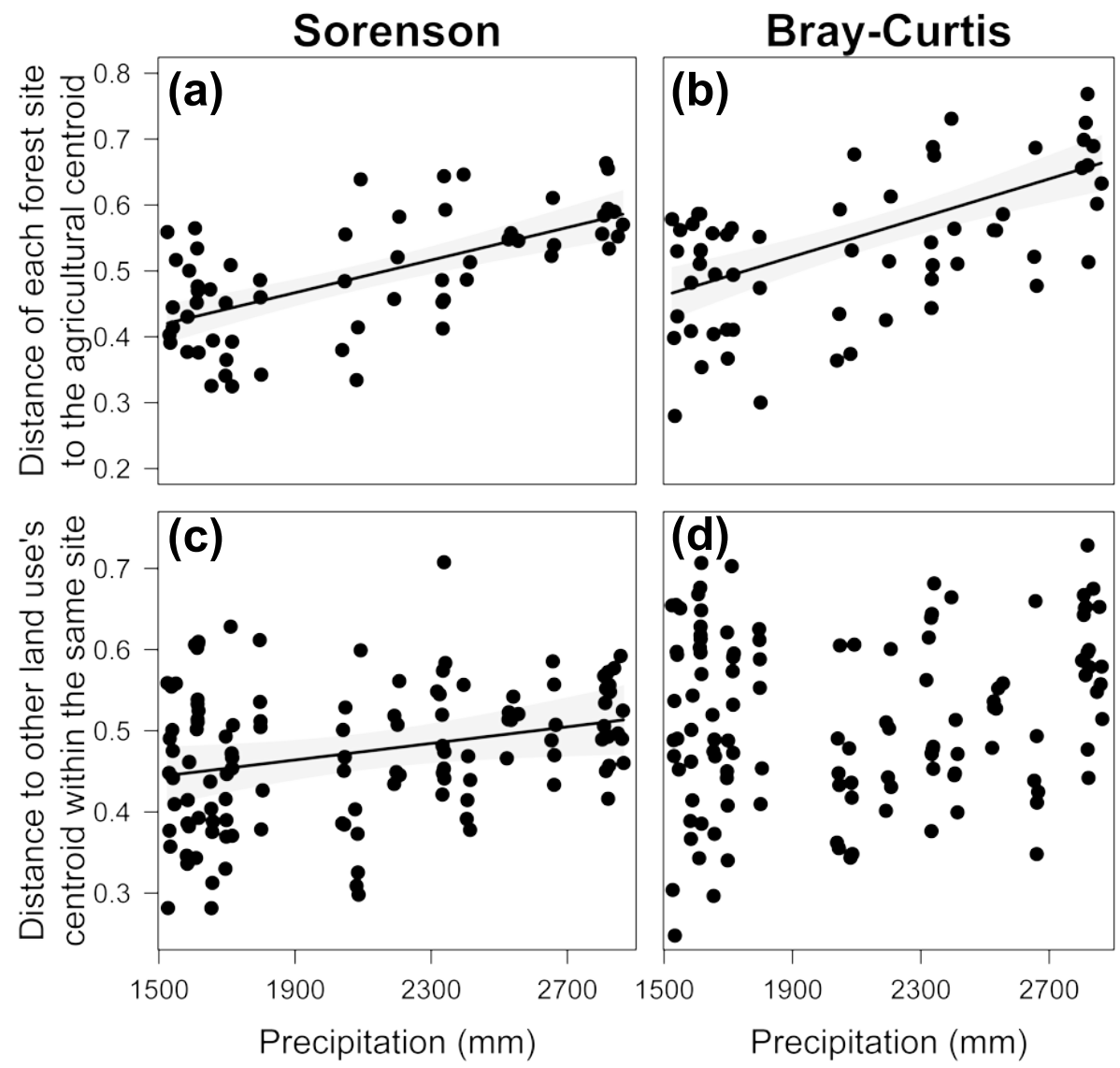

Figure 6: Agricultural communities are more similar to communities in drier than wetter

forests. In Panels A and B, each point represents the distance from a forest plot to the centroid of all agricultural plots. A greater centroid distance indicates that the site's bird community is more distinct from the bird community found in the "average" agricultural site. For all plots, lines depict significant effects of forest-site precipitation on centroid distances; shaded regions are $95 \%$ confidence intervals. Centroid distances are calculated from the turnover component of Sorenson (Panel A, C) and Bray-Curtis (Panel B, D) dissimilarity. In Panels C and D, points represent the distance of each plot to the centroid of all the plots located at the same site but in the opposite land use. Compared to drier regions, wetter regions exhibit significantly more divergence between communities in forest versus agriculture with Sorenson metrics (Panel C) and marginally more divergence for Bray-Curtis metrics (Panel D). 